\title{
Applications of Beta Negative Binomial Distribution Series on Holomorphic Functions
}

\author{
Abbas Kareem Wanas ${ }^{1}$ and Najah Ali Jiben Al-Ziadi ${ }^{2}$ \\ ${ }^{1}$ Department of Mathematics, College of Science, University of Al-Qadisiyah, Iraq \\ e-mail: abbas.kareem.w@qu.edu.iq \\ ${ }^{2}$ Department of Mathematics, College of Education, University of Al-Qadisiyah, Iraq \\ e-mail: najah.ali@qu.edu.iq
}

\begin{abstract}
The purpose of this article is to derive the necessary and sufficient conditions for the power series $\mathfrak{P} \wp_{\lambda, \gamma}^{\mu}(t)$ whose coefficients are probabilities of the beta negative binomial distribution to be in the family $\mathcal{F}(\sigma, \tau, \epsilon, \eta, \mu, \lambda, \gamma)$ of holomorphic functions which are defined in the open unit disk. We establish a number of important geometric properties, such as, coefficient estimates, extreme points, neighborhood property, integral representation, radii of starlikeness and convexity and Hadamard product properties for functions belongs to this family. Also we determinate some differential subordination properties of the power series $\mathfrak{P} \rho_{\lambda, \gamma}^{\mu}(t)$.
\end{abstract}

\section{Introduction and Preliminaries}

Indicate by $\mathcal{A}$ the family of all holomorphic functions $\mathfrak{U}$ in the open unit disk $\mathfrak{D}=\{t \in \mathbb{C}:|t|<1\}$ and having the type

$$
\mathfrak{U}(t)=t+\sum_{\mathrm{i}=2}^{\infty} a_{\mathrm{i}} t^{\mathrm{i}},
$$

Also, let $\mathcal{W}$ indicate the subfamily of $\mathcal{A}$ containing the functions of the type:

$$
\mathfrak{U}(t)=t-\sum_{\mathrm{i}=2}^{\infty} a_{\mathrm{i}} t^{\mathrm{i}} \quad\left(a_{\mathrm{i}} \geq 0\right)
$$

Received: March 5, 2021; Accepted: March 20, 2021

2010 Mathematics Subject Classification: 30C45.

Keywords and phrases: holomorphic function, beta negative binomial distribution, probability, coefficient estimates, integral representation, neighborhood, subordination. 
A function $\mathfrak{U} \in \mathcal{W}$ is said to be starlike of order $m(0 \leq m<1)$ if it fulfills the condition:

$$
\mathfrak{R}\left\{\frac{t \mathfrak{U}^{\prime}(t)}{\mathfrak{U}(t)}\right\}>m \quad(t \in \mathfrak{D})
$$

and is said to be convex of order $m(0 \leq m<1)$ if it fulfills the condition:

$$
\mathfrak{R}\left\{1+\frac{t \mathfrak{U}^{\prime \prime}(t)}{\mathfrak{U}^{\prime}(t)}\right\}>m \quad(t \in \mathfrak{D}) .
$$

Denote by $S^{*}(m)$ and $C(m)$ the families of starlike and convex functions of order $m$, respectively. These families were introduced and studied by Silverman [28].

For two functions $\mathfrak{F}$ and $\mathcal{Y}$, holomorphic in the unit disk $\mathfrak{D}$, we say that the function $\mathfrak{F}(\mathfrak{M})$ is subordinate to $\mathcal{Y}(\mathfrak{M})$ in $\mathfrak{D}$, and write

$$
\mathfrak{F}(\mathfrak{M}) \prec \mathcal{Y}(\mathfrak{B}) \quad(\mathfrak{B} \in \mathfrak{D}),
$$

if there exists a Schwarz function $\mathfrak{S}(\mathfrak{M})$, holomorphic in $\mathfrak{D}$, with

$$
\mathfrak{S}(0)=0 \text { and }|\subseteq(\mathfrak{M})|<1 \quad(\mathfrak{M} \in \mathfrak{D}) \text {, }
$$

such that

$$
\mathfrak{F}(\mathfrak{M})=\mathcal{Y}(\mathfrak{S}(\mathfrak{B})) \quad(\mathfrak{M} \in \mathfrak{D}) .
$$

In special, if the function $\mathcal{Y}$ is univalent in $\mathfrak{D}$, then

$$
\mathfrak{F}(0)=\mathcal{Y}(0) \text { and } \mathfrak{F}(\mathfrak{D}) \subset \mathcal{Y}(\mathfrak{D}) .
$$

The Hadamard product $*$ of $\mathfrak{F}$ and $\mathcal{Y}$ in $\mathcal{W}$ is defined by

$$
(\mathfrak{F} * \mathcal{Y})(t)=t-\sum_{\mathrm{i}=2}^{\infty} a_{\mathrm{i}} b_{\mathrm{i}} t^{\mathrm{i}}
$$

where

$$
\mathfrak{F}(t)=t-\sum_{\mathrm{i}=2}^{\infty} a_{\mathrm{i}} t^{\mathrm{i}} \text { and } \mathcal{y}(t)=t-\sum_{\mathrm{i}=2}^{\infty} b_{\mathrm{i}} t^{\mathrm{i}}
$$

Let $\Upsilon: \mathbb{C}^{3} \times \mathfrak{D} \rightarrow \mathbb{C}$ and let $\mathfrak{H}$ be univalent in $\mathfrak{D}$. Assume that $\mathcal{T}, \Upsilon$ are holomorphic and univalent in $\mathfrak{D}$ if $\mathcal{T}$ satisfies the differential subordination

$$
\Upsilon\left(\mathcal{T}(t), t \mathcal{T}^{\prime}(t), t^{2} \mathcal{T}^{\prime \prime}(t) ; t\right) \prec \mathfrak{H}(t),
$$


then $\mathcal{T}$ is named a solution of the differential subordination (1.3). The univalent function $\mathfrak{L}$ is named a dominant of the solution of (1.3), or further simply dominant if $\mathcal{T}<\mathfrak{L}$ for all $\mathcal{T}$ satisfying (1.3). A dominant $\widetilde{\mathfrak{L}}$ that satisfies $\widetilde{\mathfrak{L}} \prec \mathfrak{L}$ for all dominants $\mathfrak{L}$ of (1.3) is said to be the best dominant of (1.3).

In recent years, various authors investigated many interesting results in differential subordination (see for example [1,7,11,13,15,16,17,18,25,26,31,34]).

"The elementary distributions such as the Poisson, the Pascal, the Logarithmic, the Binomial, the Borel have been partially studied in the Geometric Function Theory from a theoretical point of view (see for example [2,10,23,24,33])."

A discrete random variable $x$ is said to have a beta negative binomial distribution if it takes the values $0,1,2,3, \ldots$ with the probabilities $\frac{\beta(\lambda+\mu, \gamma)}{\beta(\lambda, \gamma)}, \mu \frac{\beta(\lambda+\mu, \gamma+1)}{\beta(\lambda, \gamma)}, \frac{1}{2} \mu(\mu+$ $1 \beta \lambda+\mu, \gamma+2 \beta \lambda, \gamma, \ldots$ respectively, where $\lambda, \gamma, \mu$ are named the parameters.

$$
P(x=r)=\left(\begin{array}{c}
\mu+r-1 \\
r
\end{array}\right) \frac{\beta(\lambda+\mu, \gamma+r)}{\beta(\lambda, \gamma)}, \quad r=0,1,2,3, \ldots .
$$

We can write the above probability as follows:

$$
\begin{aligned}
P(x=r)=\left(\begin{array}{c}
\mu+r-1 \\
r
\end{array}\right) \frac{\beta(\lambda+\mu, \gamma+r)}{\beta(\lambda, \gamma)} & =\frac{\Gamma(\mu+r)}{r ! \Gamma(\mu)} \frac{\Gamma(\lambda+\mu) \Gamma(\gamma+r) \Gamma(\lambda+\gamma)}{\Gamma(\lambda+\mu+\gamma+r) \Gamma(\lambda) \Gamma(\gamma)} \\
& =\frac{(\lambda)_{\mu}(\mu)_{r}(\gamma)_{r}}{(\lambda+\gamma)_{\mu}(\mu+\lambda+\gamma)_{r} r !}
\end{aligned}
$$

where $(\delta)_{\mathrm{i}}$ is the Pochhammer symbol defined by

$$
(\delta)_{\mathrm{i}}=\frac{\Gamma(\delta+\mathrm{i})}{\Gamma(\delta)}=\left\{\begin{array}{cc}
1 & (\mathrm{i}=0) \\
\delta(\delta+1) \ldots(\delta+\mathrm{i}-1)(\mathrm{i} \in \mathbb{N})
\end{array} .\right.
$$

Now, we introduce a power series whose coefficients are probabilities of the beta negative binomial distribution, that is

$$
\mathfrak{X}_{\lambda, \gamma}^{\mu}(t)=t+\sum_{\mathrm{i}=2}^{\infty} \frac{(\lambda)_{\mu}(\mu)_{\mathrm{i}-1}(\gamma)_{\mathrm{i}-1}}{(\lambda+\gamma)_{\mu}(\mu+\lambda+\gamma)_{\mathrm{i}-1}(\mathrm{i}-1) !} t^{\mathrm{i}}, \quad t \in \mathfrak{D},
$$

where $\lambda, \gamma$ and $\mu$ are greater than zero. We see that, by using ratio test we conclude that the radius of convergence of the above power series is infinity. 
We also define the series

$$
\wp_{\lambda, \gamma}^{\mu}(t)=2 t-\mathfrak{X}_{\lambda, \gamma}^{\mu}(t)=t-\sum_{\mathrm{i}=2}^{\infty} \frac{(\lambda)_{\mu}(\mu)_{\mathrm{i}-1}(\gamma)_{\mathrm{i}-1}}{(\lambda+\gamma)_{\mu}(\mu+\lambda+\gamma)_{\mathrm{i}-1}(\mathrm{i}-1) !} t^{\mathrm{i}}, \quad t \in \mathfrak{D} .
$$

Now, we consider the linear operator $\mathfrak{P} \wp_{\lambda, \gamma}^{\mu}(t): \mathcal{W} \rightarrow \mathcal{W}$ defined by the convolution (Hadamard product)

$$
\mathfrak{B} \wp_{\lambda, \gamma}^{\mu}(t)=\wp(\mu, t) * \mathfrak{U}(t)=t-\sum_{\mathrm{i}=2}^{\infty} \frac{(\lambda)_{\mu}(\mu)_{\mathrm{i}-1}(\gamma)_{\mathrm{i}-1}}{(\lambda+\gamma)_{\mu}(\mu+\lambda+\gamma)_{\mathrm{i}-1}(\mathrm{i}-1) !} a_{\mathrm{i}} t^{\mathrm{i}}, \quad\left(a_{\mathrm{i}} \geq 0\right) .
$$

In our investigations we shall need the next lemmas:

Lemma 1.1 [22]. Let $\mathfrak{L}(t) \neq 0$ be univalent in $\mathfrak{D}$. Let $\vartheta$ and $\varphi$ be holomorphic in a domain $D$ containing $\mathfrak{L}(\mathfrak{D})$ with $\varphi(w) \neq 0$ when $w \in \mathfrak{L}(\mathfrak{D})$. Set $\mathcal{Q}(t)=$ $t \mathfrak{L}^{\prime}(t) \varphi(\mathfrak{L}(t))$ and $\mathfrak{H}(t)=\vartheta(\mathfrak{L}(t))+\mathcal{Q}(t)$. Suppose that

(1) $\mathcal{Q}(t)$ is starlike univalent in $\mathfrak{D}$,

(2) $\mathfrak{R}\left\{\frac{t \mathfrak{W}^{\prime}(t)}{Q(t)}\right\}>0$ for $t \in \mathfrak{D}$.

If $\mathcal{T}$ is holomorphic function in $\mathfrak{D}$ and

$$
\vartheta(\mathcal{T}(t))+t \mathcal{T}^{\prime}(t) \varphi(\mathcal{T}(t)) \prec \vartheta(\mathfrak{L}(t))+t \mathfrak{R}^{\prime}(t) \varphi(\mathfrak{L}(t)),
$$

then $\mathcal{T} \prec \mathfrak{L}$ and $\mathfrak{L}$ is the best dominant of (1.6).

Lemma 1.2 [9]. Let $u, v \in \mathbb{C}$ and suppose that $\mathfrak{J}$ is convex and univalent in $\mathfrak{D}$ with $\mathfrak{I}(0)=1$ and $\mathfrak{R}\{u \mathfrak{I}(t)+v\}>0,(t \in \mathfrak{D})$. If $\mathfrak{L}$ is holomorphic in $\mathfrak{D}$ with $\mathfrak{L}(0)=1$, then the subordination

$$
\mathfrak{L}(t)+\frac{t \mathfrak{L}^{\prime}(t)}{u \mathfrak{L}(t)+v} \prec \mathfrak{I}(t)
$$

implies that

$$
\mathfrak{L}(t) \prec \mathfrak{I}(t), \quad(t \in \mathfrak{D})
$$

and $\mathfrak{J}$ is the best dominant.

\section{Main Results}

We begin this section by defining the family $\mathcal{F}(\sigma, \tau, \epsilon, \eta, \mu, \lambda, \gamma)$ as follows: 
Definition 2.1. A function $\mathfrak{P} \wp_{\lambda, \gamma}^{\mu}$ is said to be in the family $\mathcal{F}(\sigma, \tau, \epsilon, \eta, \mu, \lambda, \gamma)$ if it fulfills

$$
\left|t\left(\mathfrak{P} \wp_{\lambda, \gamma}^{\mu}(t)\right)^{\prime \prime}\right|<\eta\left|\sigma t\left(\mathfrak{P} \wp_{\lambda, \gamma}^{\mu}(t)\right)^{\prime \prime}+(\tau-\epsilon+\sigma)\left(\mathfrak{P} \wp_{\lambda, \gamma}^{\mu}(t)\right)^{\prime}\right|,
$$

where $0 \leq \sigma<1,0<\tau \leq 1,0 \leq \epsilon<1,0<\eta<1$ and $t \in \mathfrak{D}$.

Such type of study was carried out by numerous researchers for another families (see for example $[3,4,5,6,8,14,19,20,21,29,30,32,35])$.

In the first theorem, we establish the necessary and sufficient conditions of the power series $\mathfrak{P} \wp_{\lambda, \gamma}^{\mu}$ to be in the family $\mathcal{F}(\sigma, \tau, \epsilon, \eta, \mu, \lambda, \gamma)$.

Theorem 2.1. A function $\mathfrak{P} \wp_{\lambda, \gamma}^{\mu}$ is in the family $\mathcal{F}(\sigma, \tau, \epsilon, \eta, \mu, \lambda, \gamma)$ if and only if

$$
\sum_{\mathrm{i}=2}^{\infty} \frac{\mathrm{i}(\lambda)_{\mu}(\mu)_{\mathrm{i}-1}(\gamma)_{\mathrm{i}-1}[\mathrm{i}(\sigma \eta+1)+\eta(\tau-\epsilon)-1]}{(\lambda+\gamma)_{\mu}(\mu+\lambda+\gamma)_{\mathrm{i}-1}(\mathrm{j}-1) !} a_{\mathrm{i}} \leq \eta(\tau-\epsilon+\sigma),
$$

where $0 \leq \sigma<1,0<\tau \leq 1,0 \leq \epsilon<1,0<\eta<1, \lambda, \gamma, \mu>0$ and $t \in \mathfrak{D}$.

The result is sharp for $\mathfrak{P} \wp_{\lambda, \gamma}^{\mu}$ given by

$$
\mathfrak{P} \wp_{\lambda, \gamma}^{\mu}(t)=t-\frac{\eta(\tau-\epsilon+\sigma)(\lambda+\gamma)_{\mu}(\mu+\lambda+\gamma)_{\mathrm{i}-1}(\mathfrak{j}-1) !}{\mathrm{j}(\lambda)_{\mu}(\mu)_{\mathrm{i}-1}(\gamma)_{\mathrm{i}-1}[\mathrm{j}(\sigma \eta+1)+\eta(\tau-\epsilon)-1]} t^{\mathrm{j}}, \quad(\mathrm{j} \geq 2) .
$$

Proof. Let us the inequality (2.2) holds true $|t|=1$. Then, we conclude that

$$
\begin{aligned}
& \left|t\left(\mathfrak{P} \rho_{\lambda, \gamma}^{\mu}(t)\right)^{\prime \prime}\right|-\eta\left|\sigma t\left(\mathfrak{P} \rho_{\lambda, \gamma}^{\mu}(t)\right)^{\prime \prime}+(\tau-\epsilon+\sigma)\left(\mathfrak{P} \wp_{\lambda, \gamma}^{\mu}(t)\right)^{\prime}\right| \\
= & \left|-\sum_{\mathrm{i}=2}^{\infty} \frac{\mathrm{i}(\mathrm{i}-1)(\lambda)_{\mu}(\mu)_{\mathrm{i}-1}(\gamma)_{\mathrm{i}-1}}{(\lambda+\gamma)_{\mu}(\mu+\lambda+\gamma)_{\mathrm{i}-1}(\mathrm{j}-1) !} a_{\mathrm{i}} t^{\mathrm{i}-1}\right| \\
& -\left|\eta(\tau-\epsilon+\sigma)-\sum_{\mathrm{i}=2}^{\infty} \frac{\eta \mathrm{i}(\tau-\epsilon+\sigma \mathrm{i})(\lambda)_{\mu}(\mu)_{\mathrm{i}-1}(\gamma)_{\mathrm{i}-1}}{(\lambda+\gamma)_{\mu}(\mu+\lambda+\gamma)_{\mathrm{i}-1}(\mathrm{i}-1) !} a_{\mathrm{i}} t^{\mathrm{i}-1}\right| \\
\leq & \sum_{\mathrm{i}=2}^{\infty} \frac{\mathrm{i}(\mathrm{i}-1)(\lambda)_{\mu}(\mu)_{\mathrm{i}-1}(\gamma)_{\mathrm{i}-1}}{(\lambda+\gamma)_{\mu}(\mu+\lambda+\gamma)_{\mathrm{i}-1}(\mathrm{i}-1) !} a_{\mathrm{i}}|t|^{\mathrm{i}-1}-\eta(\tau-\epsilon+\sigma) \\
& +\sum_{\mathrm{i}=2}^{\infty} \frac{\eta \mathrm{i}(\tau-\epsilon+\sigma \mathrm{i})(\lambda)_{\mu}(\mu)_{\mathrm{i}-1}(\gamma)_{\mathrm{i}-1}}{(\lambda+\gamma)_{\mu}(\mu+\lambda+\gamma)_{\mathrm{i}-1}(\mathrm{i}-1) !} a_{\mathrm{i}}|t|^{\mathrm{i}-1}
\end{aligned}
$$


$=\sum_{\mathrm{i}=2}^{\infty} \frac{\mathrm{i}(\lambda)_{\mu}(\mu)_{\mathrm{i}-1}(\gamma)_{\mathrm{i}-1}[\mathrm{i}(\sigma \eta+1)+\eta(\tau-\epsilon)-1]}{(\lambda+\gamma)_{\mu}(\mu+\lambda+\gamma)_{\mathrm{i}-1}(\mathrm{i}-1) !} a_{\mathrm{i}}-\eta(\tau-\epsilon+\sigma) \leq 0$,

by hypothesis. Hence, by maximum modulus principle, we obtain $\mathfrak{P} \wp_{\lambda, \gamma}^{\mu} \in$ $\mathcal{F}(\sigma, \tau, \epsilon, \eta, \mu, \lambda, \gamma)$.

Conversely, let $\mathfrak{P} \wp_{\lambda, \gamma}^{\mu} \in \mathcal{F}(\sigma, \tau, \epsilon, \eta, \mu, \lambda, \gamma)$. Then from (2.2), we obtain

$$
\begin{aligned}
& \left|t\left(\mathfrak{P} \wp_{\lambda, \gamma}^{\mu}(t)\right)^{\prime \prime}\right|-\eta\left|\sigma t\left(\mathfrak{P} \wp_{\lambda, \gamma}^{\mu}(t)\right)^{\prime \prime}+(\tau-\epsilon+\sigma)\left(\mathfrak{P} \wp_{\lambda, \gamma}^{\mu}(t)\right)^{\prime}\right| \\
& \left|\frac{t\left(\mathfrak{P} \rho_{\lambda, \gamma}^{\mu}(t)\right)^{\prime \prime}}{\sigma t\left(\mathfrak{P} \rho_{\lambda, \gamma}^{\mu}(t)\right)^{\prime \prime}+(\tau-\epsilon+\sigma)\left(\mathfrak{P} \wp_{\lambda, \gamma}^{\mu}(t)\right)^{\prime}}\right| \\
& =\left|\frac{\sum_{\mathrm{j}=2}^{\infty} \frac{\mathrm{i}(\mathrm{i}-1)(\lambda)_{\mu}(\mu)_{\mathrm{i}-1}(\gamma)_{\mathrm{i}-1}}{(\lambda+\gamma)_{\mu}(\mu+\lambda+\gamma)_{\mathrm{i}-1}(\mathrm{i}-1) !} a_{\mathrm{i}} t^{\mathrm{i}-1}}{(\tau-\epsilon+\sigma)-\sum_{\mathrm{j}=2}^{\infty} \frac{\mathrm{i}(\tau-\epsilon+\sigma \mathrm{i})(\lambda)_{\mu}(\mu)_{\mathrm{i}-1}(\gamma)_{\mathrm{i}-1}}{(\lambda+\gamma)_{\mu}(\mu+\lambda+\gamma)_{\mathrm{i}-1}(\mathrm{i}-1) !} a_{\mathrm{i}} t^{\mathrm{i}-1}}\right|<\eta .
\end{aligned}
$$

Since $\mathfrak{R}(t) \leq|t|$ for all $t(t \in \mathfrak{D})$, we get

$$
\Re\left\{\frac{\sum_{\mathrm{j}=2}^{\infty} \frac{\mathrm{i}(\mathrm{i}-1)(\lambda)_{\mu}(\mu)_{\mathrm{i}-1}(\gamma)_{\mathrm{i}-1}}{(\lambda+\gamma)_{\mu}(\mu+\lambda+\gamma)_{\mathrm{i}-1}(\mathrm{i}-1) !} a_{\mathrm{i}} t^{\mathrm{i}-1}}{(\lambda+1)(1-\sigma)-\sum_{\mathrm{j}=2}^{\infty} \frac{\mathrm{i}(\tau-\epsilon+\sigma \mathrm{j})(\lambda)_{\mu}(\mu)_{\mathrm{i}-1}(\gamma)_{\mathrm{i}-1}}{(\lambda+\gamma)_{\mu}(\mu+\lambda+\gamma)_{\mathrm{i}-1}(\mathrm{i}-1) !} a_{\mathrm{i}} t^{\mathrm{i}-1}}\right\}<\eta .
$$

We select the value of $t$ on the real axis hence $\frac{t\left(\mathfrak{B} \delta_{\lambda, \gamma}^{\mu}(t)\right)^{\prime \prime}}{\left(\mathfrak{B} \delta_{\lambda, \gamma}^{\mu}(t)\right)^{\prime}}$ is real. Upon clearing the denominator of (2.4) and putting $t \rightarrow 1^{-}$, through real values, thus we can write (2.4) as

$$
\sum_{\mathrm{i}=2}^{\infty} \frac{\mathrm{i}(\lambda)_{\mu}(\mu)_{\mathrm{i}-1}(\gamma)_{\mathrm{i}-1}[\mathrm{i}(\sigma \eta+1)+\eta(\tau-\epsilon)-1]}{(\lambda+\gamma)_{\mu}(\mu+\lambda+\gamma)_{\mathrm{i}-1}(\mathrm{i}-1) !} a_{\mathrm{i}} \leq \eta(\tau-\epsilon+\sigma),
$$

which completes the proof.

Corollary 2.1. If $\mathfrak{P} \rho_{\lambda, \gamma}^{\mu} \in \mathcal{F}(\sigma, \tau, \epsilon, \eta, \mu, \lambda, \gamma)$, then 


$$
a_{\mathrm{i}} \leq \frac{\eta(\tau-\epsilon+\sigma)(\lambda+\gamma)_{\mu}(\mu+\lambda+\gamma)_{\mathrm{i}-1}(\mathrm{j}-1) !}{\mathrm{j}(\lambda)_{\mu}(\mu)_{\mathrm{i}-1}(\gamma)_{\mathrm{i}-1}[\mathrm{i}(\sigma \eta+1)+\eta(\tau-\epsilon)-1]}, \quad(\mathrm{j} \geq 2) .
$$

Next, we obtain an extreme points of the family $\mathfrak{P} \wp_{\lambda, \gamma}^{\mu} \in \mathcal{F}(\sigma, \tau, \epsilon, \eta, \mu, \lambda, \gamma)$.

Theorem 2.2. Suppose that $\mathfrak{P} \rho_{\lambda, \gamma_{1}}^{\mu}(t)=t$ and

$$
\mathfrak{P} \wp_{\lambda, \gamma_{\mathrm{i}}}^{\mu}(t)=t-\frac{\eta(\tau-\epsilon+\sigma)(\lambda+\gamma)_{\mu}(\mu+\lambda+\gamma)_{\mathrm{i}-1}(\mathrm{i}-1) !}{\mathrm{i}(\lambda)_{\mu}(\mu)_{\mathrm{i}-1}(\gamma)_{\mathrm{i}-1}[\mathrm{i}(\sigma \eta+1)+\eta(\tau-\epsilon)-1]} t^{\mathrm{i}} \quad, \quad(\mathrm{j} \geq 2) .
$$

Then $\mathfrak{P} \wp_{\lambda, \gamma}^{\mu} \in \mathcal{F}(\sigma, \tau, \epsilon, \eta, \mu, \lambda, \gamma)$ if and only if it can be expressed in the shape

$$
\mathfrak{P} \wp_{\lambda, \gamma}^{\mu}(t)=\sum_{\mathrm{i}=1}^{\infty} \vartheta_{\mathrm{i}} B_{\mu_{\mathrm{i}}}(t),
$$

where $\vartheta_{\mathrm{i}} \geq 0, \mathrm{i} \geq 1$ and $\sum_{\mathrm{i}=1}^{\infty} \vartheta_{\mathrm{i}}=1$.

Proof. Suppose that $\mathfrak{P} \wp_{\lambda, \gamma}^{\mu}$ is expressed in the shape (2.5). Therefore

$$
\begin{aligned}
\mathfrak{P} \wp_{\lambda, \gamma}^{\mu}(t)= & \sum_{\mathrm{i}=1}^{\infty} \vartheta_{\mathrm{i}} \mathfrak{P} \wp_{\lambda, \gamma_{\mathrm{i}}}^{\mu}(t)=\vartheta_{1} \mathfrak{P} \wp_{\lambda, \gamma_{1}}^{\mu}(t)+\sum_{\mathrm{i}=2}^{\infty} \vartheta_{\mathrm{i}} \mathfrak{P} \wp_{\lambda, \gamma_{\mathrm{i}}}^{\mu}(t) \\
= & \left(1-\sum_{\mathrm{i}=2}^{\infty} \vartheta_{\mathrm{i}}\right) t \\
& +\sum_{\mathrm{i}=2}^{\infty} \vartheta_{\mathrm{i}}\left(t-\frac{\eta(\tau-\epsilon+\sigma)(\lambda+\gamma)_{\mu}(\mu+\lambda+\gamma)_{\mathrm{i}-1}(\mathrm{i}-1) !}{\mathrm{i}(\lambda)_{\mu}(\mu)_{\mathrm{i}-1}(\gamma)_{\mathrm{i}-1}[\mathrm{i}(\sigma \eta+1)+\eta(\tau-\epsilon)-1]} t^{\mathrm{i}}\right) \\
= & t-\sum_{\mathrm{i}=2}^{\infty} \frac{\eta(\tau-\epsilon+\sigma)(\lambda+\gamma)_{\mu}(\mu+\lambda+\gamma)_{\mathrm{i}-1}(\mathrm{i}-1) !}{\mathrm{i}(\lambda)_{\mu}(\mu)_{\mathrm{i}-1}(\gamma)_{\mathrm{i}-1}[\mathrm{i}(\sigma \eta+1)+\eta(\tau-\epsilon)-1]} \vartheta_{\mathrm{i}} .
\end{aligned}
$$

Now

$$
\begin{aligned}
& \sum_{\mathrm{i}=2}^{\infty} \frac{\mathrm{i}(\lambda)_{\mu}(\mu)_{\mathrm{i}-1}(\gamma)_{\mathrm{i}-1}[\mathrm{i}(\sigma \eta+1)+\eta(\tau-\epsilon)-1]}{\eta(\tau-\epsilon+\sigma)(\lambda+\gamma)_{\mu}(\mu+\lambda+\gamma)_{\mathrm{i}-1}(\mathrm{i}-1) !} \times \\
& \times \frac{\eta(\tau-\epsilon+\sigma)(\lambda+\gamma)_{\mu}(\mu+\lambda+\gamma)_{\mathrm{i}-1}(\mathrm{i}-1) !}{\mathrm{i}(\lambda)_{\mu}(\mu)_{\mathrm{i}-1}(\gamma)_{\mathrm{i}-1}[\mathrm{i}(\sigma \eta+1)+\eta(\tau-\epsilon)-1]} \vartheta_{\mathrm{i}}
\end{aligned}
$$




$$
=\sum_{\mathrm{i}=2}^{\infty} \vartheta_{\mathrm{i}}=1-\vartheta_{1} \leq 1
$$

This shows that $\mathfrak{P} \wp_{\lambda, \gamma}^{\mu} \in \mathcal{F}(\sigma, \tau, \epsilon, \eta, \mu, \lambda, \gamma)$.

Conversely, assume that $\mathfrak{P} \wp_{\lambda, \gamma}^{\mu}$ given by (1.5) be in the family $\mathcal{F}(\sigma, \tau, \epsilon, \eta, \mu, \lambda, \gamma)$. Then by Corollary 2.1, we find that

$$
a_{\mathrm{i}} \leq \frac{\eta(\tau-\epsilon+\sigma)(\lambda+\gamma)_{\mu}(\mu+\lambda+\gamma)_{\mathrm{i}-1}(\mathrm{i}-1) !}{\mathrm{i}(\lambda)_{\mu}(\mu)_{\mathrm{i}-1}(\gamma)_{\mathrm{i}-1}[\mathrm{i}(\sigma \eta+1)+\eta(\tau-\epsilon)-1]} .
$$

we can set

$$
\vartheta_{\mathrm{i}}=\frac{\mathrm{i}\left((\lambda)_{\mu}\right)^{2}\left((\mu)_{\mathrm{i}-1}\right)^{2}\left((\gamma)_{\mathrm{i}-1}\right)^{2}[\mathrm{i}(\sigma \eta+1)+\eta(\tau-\epsilon)-1]}{\eta(\tau-\epsilon+\sigma)\left((\lambda+\gamma)_{\mu}\right)^{2}\left((\mu+\lambda+\gamma)_{\mathrm{i}-1}\right)^{2}((\mathrm{i}-1) !)^{2}} a_{\mathrm{i}} \quad(\mathrm{i} \geq 2),
$$

where $\vartheta_{1}=1-\sum_{\mathrm{i}=2}^{\infty} \vartheta_{\mathrm{i}}$. Thus

$$
\begin{aligned}
\mathfrak{P} \wp_{\lambda, \gamma}^{\mu}(t)= & t-\sum_{\mathrm{i}=2}^{\infty} \frac{(\lambda)_{\mu}(\mu)_{\mathrm{i}-1}(\gamma)_{\mathrm{i}-1}}{(\lambda+\gamma)_{\mu}(\mu+\lambda+\gamma)_{\mathrm{i}-1}(\mathrm{i}-1) !} a_{\mathrm{i}} t^{\mathrm{i}} \\
= & t-\sum_{\mathrm{i}=2}^{\infty} \frac{(\lambda)_{\mu}(\mu)_{\mathrm{i}-1}(\gamma)_{\mathrm{i}-1}}{(\lambda+\gamma)_{\mu}(\mu+\lambda+\gamma)_{\mathrm{i}-1}(\mathrm{i}-1) !} \times \\
& \times \frac{\eta(\tau-\epsilon+\sigma)\left((\lambda+\gamma)_{\mu}\right)^{2}\left((\mu+\lambda+\gamma)_{\mathrm{i}-1}\right)^{2}((\mathrm{i}-1) !)^{2}}{\mathrm{i}\left((\lambda)_{\mu}\right)^{2}\left((\mu)_{\mathrm{i}-1}\right)^{2}\left((\gamma)_{\mathrm{i}-1}\right)^{2}[\mathrm{i}(\sigma \eta+1)+\eta(\tau-\epsilon)-1]} \vartheta_{\mathrm{i}} t^{\mathrm{i}} \\
= & t-\sum_{\mathrm{i}=2}^{\infty} \frac{\eta(\tau-\epsilon+\sigma)(\lambda+\gamma)_{\mu}(\mu+\lambda+\gamma)_{\mathrm{i}-1}(\mathrm{i}-1) !}{\mathrm{i}(\lambda)_{\mu}(\mu)_{\mathrm{i}-1}(\gamma)_{\mathrm{i}-1}[\mathrm{i}(\sigma \eta+1)+\eta(\tau-\epsilon)-1]} \vartheta_{\mathrm{i}} t^{\mathrm{i}} \\
= & t-\sum_{\mathrm{i}=2}^{\infty}\left(t-\mathfrak{B} \rho_{\lambda, \gamma_{\mathrm{j}}}^{\mu}(t)\right) \vartheta_{\mathrm{i}} \\
= & \left(1-\sum_{\mathrm{i}=2}^{\infty} \vartheta_{\mathrm{i}} t+\sum_{\mathrm{i}=2}^{\infty} \vartheta_{\mathrm{i}} \mathfrak{P} \delta_{\lambda, \gamma_{\mathrm{i}}}^{\mu}(t)\right.
\end{aligned}
$$




$$
=\vartheta_{1} \mathfrak{P} \wp_{\lambda, \gamma_{1}}^{\mu}(t)+\sum_{\mathrm{i}=2}^{\infty} \vartheta_{\mathrm{i}} \mathfrak{P} \wp_{\lambda, \gamma_{\mathrm{j}}}^{\mu}(t)=\sum_{\mathrm{i}=1}^{\infty} \vartheta_{\mathrm{i}} \mathfrak{P} \wp_{\lambda, \gamma_{\mathrm{i}}}^{\mu}(t),
$$

that is the required representation.

In view of the work of Goodman [12] and Ruscheweyh [27], we define the $\rho$-neighborhood for the power series $\mathfrak{P} \wp_{\lambda, \gamma}^{\mu}$ by the definition below:

$$
\begin{aligned}
N_{\rho}\left(\mathfrak{P} \rho_{\lambda, \gamma}^{\mu}\right)= & \left\{h_{\lambda, \gamma}^{\mu}: h_{\lambda, \gamma}^{\mu}(t)=t-\sum_{\mathrm{i}=2}^{\infty} \frac{(\lambda)_{\mu}(\mu)_{\mathrm{i}-1}(\gamma)_{\mathrm{i}-1}}{(\lambda+\gamma)_{\mu}(\mu+\lambda+\gamma)_{\mathrm{i}-1}(\mathrm{j}-1) !} b_{\mathrm{i}} t^{\mathrm{i}}\right. \\
& \text { and } \left.\sum_{\mathrm{i}=2}^{\infty} \frac{\mathrm{j}(\lambda)_{\mu}(\mu)_{\mathrm{i}-1}(\gamma)_{\mathrm{i}-1}}{(\lambda+\gamma)_{\mu}(\mu+\lambda+\gamma)_{\mathrm{i}-1}(\mathrm{i}-1) !}\left|a_{\mathrm{i}}-b_{\mathrm{i}}\right| \leq \rho, 0 \leq \rho<1\right\} .
\end{aligned}
$$

In particular, for the identity function $I(t)=t$, we have

$$
\begin{aligned}
N_{\rho}\left(I_{\lambda, \gamma}^{\mu}\right)=\left\{h_{\lambda, \gamma}^{\mu}:\right. & h_{\lambda, \gamma}^{\mu}(t)=t-\sum_{\mathrm{i}=2}^{\infty} \frac{(\lambda)_{\mu}(\mu)_{\mathrm{i}-1}(\gamma)_{\mathrm{i}-1}}{(\lambda+\gamma)_{\mu}(\mu+\lambda+\gamma)_{\mathrm{i}-1}(\mathrm{i}-1) !} b_{\mathrm{i}} t^{\mathrm{i}} \\
& \text { and } \left.\sum_{\mathrm{i}=2}^{\infty} \frac{\mathrm{i}(\lambda)_{\mu}(\mu)_{\mathrm{i}-1}(\gamma)_{\mathrm{i}-1}}{(\lambda+\gamma)_{\mu}(\mu+\lambda+\gamma)_{\mathrm{i}-1}(\mathrm{j}-1) !}\left|b_{\mathrm{i}}\right| \leq \rho, 0 \leq \rho<1\right\} .
\end{aligned}
$$

Definition 2.2. A function $\mathfrak{P} \wp_{\lambda, \gamma}^{\mu}$ is called in the family $\mathcal{F}_{3}(\sigma, \tau, \epsilon, \eta, \mu, \lambda, \gamma)$ if there is a function $h_{\lambda, \gamma}^{\mu} \in \mathcal{F}(\sigma, \tau, \epsilon, \eta, \mu, \lambda, \gamma)$, such that

$$
\left|\frac{\mathfrak{P} \rho_{\lambda, \gamma}^{\mu}(t)}{h_{\lambda, \gamma}^{\mu}(t)}-1\right|<1-3(t \in \mathfrak{D}, 0 \leq 3<1) .
$$

Theorem 2.3. If $h_{\lambda, \gamma}^{\mu} \in \mathcal{F}(\sigma, \tau, \epsilon, \eta, \mu, \lambda, \gamma)$ and

$$
3=1-\frac{\rho[2(\sigma \eta+1)+\eta(\tau-\epsilon)-1]}{2[2(\sigma \eta+1)+\eta(\tau-\epsilon)-1]-\eta(\tau-\epsilon+\sigma)},
$$

then $N_{\rho}\left(h_{\lambda, \gamma}^{\mu}\right) \subset \mathcal{F}_{3}(\sigma, \tau, \epsilon, \eta, \mu, \lambda, \gamma)$.

Proof. Assume that $\mathfrak{P} \wp_{\lambda, \gamma}^{\mu} \in N_{\rho}\left(h_{\lambda, \gamma}^{\mu}\right)$. Then we deduce from (2.6) that 


$$
\sum_{\mathrm{i}=2}^{\infty} \frac{\mathrm{i}(\lambda)_{\mu}(\mu)_{\mathrm{i}-1}(\gamma)_{\mathrm{i}-1}}{(\lambda+\gamma)_{\mu}(\mu+\lambda+\gamma)_{\mathrm{i}-1}(\mathrm{i}-1) !}\left|a_{\mathrm{i}}-b_{\mathrm{i}}\right| \leq \rho,
$$

which implies

$$
\sum_{\mathrm{i}=2}^{\infty} \frac{(\lambda)_{\mu}(\mu)_{\mathrm{i}-1}(\gamma)_{\mathrm{i}-1}}{(\lambda+\gamma)_{\mu}(\mu+\lambda+\gamma)_{\mathrm{i}-1}(\mathrm{i}-1) !}\left|a_{\mathrm{i}}-b_{\mathrm{i}}\right| \leq \frac{\rho}{2} .
$$

Since $h_{\lambda, \gamma}^{\mu} \in \mathcal{F}(\sigma, \tau, \epsilon, \eta, \mu, \lambda, \gamma)$, by using Theorem 2.1, we have

$$
\sum_{\mathrm{i}=2}^{\infty} \frac{(\lambda)_{\mu}(\mu)_{\mathrm{i}-1}(\gamma)_{\mathrm{i}-1}}{(\lambda+\gamma)_{\mu}(\mu+\lambda+\gamma)_{\mathrm{i}-1}(\mathrm{i}-1) !} b_{\mathrm{i}} \leq \frac{\eta(\tau-\epsilon+\sigma)}{2[2(\sigma \eta+1)+\eta(\tau-\epsilon)-1]} .
$$

So that

$$
\begin{aligned}
\left|\frac{\mathfrak{P} \wp_{\lambda, \gamma}^{\mu}(t)}{h_{\lambda, \gamma}^{\mu}(t)}-1\right| \leq & \frac{\sum_{\mathrm{j}=2}^{\infty} \frac{(\lambda)_{\mu}(\mu)_{\mathrm{i}-1}(\gamma)_{\mathrm{i}-1}}{(\lambda+\gamma)_{\mu}(\mu+\lambda+\gamma)_{\mathrm{i}-1}(\mathrm{i}-1) !}\left|a_{\mathrm{i}}-b_{\mathrm{i}}\right||t|^{\mathrm{i}-1}}{1-\sum_{\mathrm{j}=2}^{\infty} \frac{(\lambda)_{\mu}(\mu)_{\mathrm{i}-1}(\gamma)_{\mathrm{i}-1}}{(\lambda+\gamma)_{\mu}(\mu+\lambda+\gamma)_{\mathrm{i}-1}(\mathrm{i}-1) !} b_{\mathrm{i}}|t|^{\mathrm{i}-1}} \\
& <\frac{\sum_{\mathrm{j}=2}^{\infty} \frac{(\lambda)_{\mu}(\mu)_{\mathrm{i}-1}(\gamma)_{\mathrm{i}-1}}{(\lambda+\gamma)_{\mu}(\mu+\lambda+\gamma)_{\mathrm{i}-1}(\mathrm{i}-1) !}\left|a_{\mathrm{i}}-b_{\mathrm{i}}\right|}{1-\sum_{\mathrm{j}=2}^{\infty} \frac{(\lambda)_{\mu}(\mu)_{\mathrm{i}-1}(\gamma)_{\mathrm{i}-1}}{(\lambda+\gamma)_{\mu}(\mu+\lambda+\gamma)_{\mathrm{i}-1}(\mathrm{i}-1) !} b_{\mathrm{i}}} \\
& \leq \frac{\rho[2(\sigma \eta+1)+\eta(\tau-\epsilon)-1]}{2[2(\sigma \eta+1)+\eta(\tau-\epsilon)-1]-\eta(\tau-\epsilon+\sigma)} \\
& =1-3 .
\end{aligned}
$$

Hence, by Definition 2.2, equivalently to $\mathfrak{P} \wp_{\lambda, \gamma}^{\mu} \in \mathcal{F}_{3}(\sigma, \tau, \epsilon, \eta, \mu, \lambda, \gamma)$ for 3 given by (2.7). This completes the proof of the theorem.

In the next theorem, we find integral representation of the power series $\mathfrak{P} \wp_{\lambda, \gamma}^{\mu}$.

Theorem 2.4. Let $\mathfrak{P} \wp_{\lambda, \gamma}^{\mu} \in \mathcal{F}(\sigma, \tau, \epsilon, \eta, \mu, \lambda, \gamma)$. Then

$$
\mathfrak{P} \wp_{\lambda, \gamma}^{\mu}(t)=\int_{0}^{t} \operatorname{ex\mathcal {T}}\left[\int_{0}^{t} \frac{\eta(\tau-\epsilon+\sigma) \psi\left(t_{1}\right)}{t_{1}\left(1-\eta \sigma \psi\left(t_{1}\right)\right)} d t_{1}\right] d t_{2},
$$

where $|\psi(t)|<1, t \in \mathfrak{D}$. 
Proof. By letting $\frac{t\left(\mathfrak{B} \delta_{\lambda, \gamma}^{\mu}(t)\right)^{\prime \prime}}{\left(\mathfrak{P} \delta_{\lambda, \gamma}^{\mu}(t)\right)^{\prime}}=E(t)$ in (2.1), we have

$$
\left|\frac{E(t)}{\sigma E(t)+\tau-\epsilon+\sigma}\right|<\eta,
$$

or equivalently

$$
\frac{E(t)}{\sigma E(t)+\tau-\epsilon+\sigma}=\eta \psi(t), \quad(|\psi(t)|<1, t \in \mathfrak{D})
$$

So

$$
\frac{\left(\mathfrak{P} \rho_{\lambda, \gamma}^{\mu}(t)\right)^{\prime \prime}}{\left(\mathfrak{P} \wp_{\lambda, \gamma}^{\mu}(t)\right)^{\prime}}=\frac{\eta(\tau-\epsilon+\sigma) \psi(t)}{t(1-\eta \sigma \psi(t))},
$$

after integration, we obtain

$$
\log \left(\left(\mathfrak{P} \wp_{\lambda, \gamma}^{\mu}(t)\right)^{\prime}\right)=\int_{0}^{t} \frac{\eta(\tau-\epsilon+\sigma) \psi(t)}{t(1-\eta \sigma \psi(t))} d t .
$$

Therefore

$$
\left(\mathfrak{P} \wp_{\lambda, \gamma}^{\mu}(t)\right)^{\prime}=\operatorname{ex\mathcal {T}}\left[\int_{0}^{t} \frac{\eta(\tau-\epsilon+\sigma) \psi(t)}{t(1-\eta \sigma \psi(t))} d t\right] .
$$

By integration once again, we get

$$
\mathfrak{P} \wp_{\lambda, \gamma}^{\mu}(t)=\int_{0}^{t} \operatorname{ex\mathcal {T}}\left[\int_{0}^{t} \frac{\eta(\tau-\epsilon+\sigma) \psi\left(t_{1}\right)}{t_{1}\left(1-\eta \sigma \psi\left(t_{1}\right)\right)} d t_{1}\right] d t_{2},
$$

and this gives the required result.

Theorem 2.5. If $\mathfrak{P} \wp_{\lambda, \gamma}^{\mu} \in \mathcal{F}(\sigma, \tau, \epsilon, \eta, \mu, \lambda, \gamma)$, then $\mathfrak{P} \wp_{\lambda, \gamma}^{\mu}$ will be starlike of order $m(0 \leq m<1)$ in the disk $|t|<r_{1}$, where

$$
r_{1}=\inf _{\dot{i}}\left\{\frac{\mathrm{i}(1-m)[\mathrm{i}(\sigma \eta+1)+\eta(\tau-\epsilon)-1]}{\eta(\mathrm{i}-m)(\tau-\epsilon+\sigma)}\right\}^{\frac{1}{\mathrm{i}-1}}, \quad(\mathrm{i} \geq 2) .
$$

Proof. It is enough to show that

$$
\left|\frac{t\left(\mathfrak{P} \wp_{\lambda, \gamma}^{\mu}(t)\right)^{\prime}}{\mathfrak{P} \wp_{\lambda, \gamma}^{\mu}(t)}-1\right| \leq 1-m \quad \text { for }|t|<r_{1} .
$$


We have

$$
\left|\frac{t\left(\mathfrak{P} \rho_{\lambda, \gamma}^{\mu}(t)\right)^{\prime}}{\mathfrak{P} \rho_{\lambda, \gamma}^{\mu}(t)}-1\right| \leq \frac{\sum_{\mathrm{j}=2}^{\infty} \frac{(\mathrm{i}-1)(\lambda)_{\mu}(\mu)_{\mathrm{i}-1}(\gamma)_{\mathrm{i}-1}}{(\lambda+\gamma)_{\mu}(\mu+\lambda+\gamma)_{\mathrm{i}-1}(\mathrm{i}-1) !} a_{\mathrm{i}}|t|^{\mathrm{i}-1}}{1-\sum_{\mathrm{j}=2}^{\infty} \frac{(\lambda)_{\mu}(\mu)_{\mathrm{i}-1}(\gamma)_{\mathrm{i}-1}}{(\lambda+\gamma)_{\mu}(\mu+\lambda+\gamma)_{\mathrm{i}-1}(\mathrm{i}-1) !} a_{\mathrm{i}}|t|^{\mathrm{i}-1}}
$$

Thus (2.8) will be satisfied if

$$
\sum_{\mathrm{i}=2}^{\infty} \frac{(\mathrm{i}-m)(\lambda)_{\mu}(\mu)_{\mathrm{i}-1}(\gamma)_{\mathrm{i}-1}}{(1-m)(\lambda+\gamma)_{\mu}(\mu+\lambda+\gamma)_{\mathrm{i}-1}(\mathrm{i}-1) !} a_{\mathrm{i}}|t|^{\mathrm{i}-1} \leq 1 .
$$

Also from Theorem 2.1, if $\mathfrak{P} \wp_{\lambda, \gamma}^{\mu} \in \mathcal{H}(\lambda, \sigma, \delta, \mu)$, then

$$
\sum_{\mathrm{i}=2}^{\infty} \frac{\mathrm{i}(\lambda)_{\mu}(\mu)_{\mathrm{i}-1}(\gamma)_{\mathrm{i}-1}[\mathrm{i}(\sigma \eta+1)+\eta(\tau-\epsilon)-1]}{\eta(\tau-\epsilon+\sigma)(\lambda+\gamma)_{\mu}(\mu+\lambda+\gamma)_{\mathrm{i}-1}(\mathrm{j}-1) !} a_{\mathrm{i}} \leq 1 .
$$

In view of (2.10), we notice that (2.9) holds true if

$$
\begin{aligned}
& \frac{(\mathfrak{j}-m)(\lambda)_{\mu}(\mu)_{\mathrm{i}-1}(\gamma)_{\mathrm{i}-1}}{(1-m)(\lambda+\gamma)_{\mu}(\mu+\lambda+\gamma)_{\mathrm{i}-1}(\mathrm{i}-1) !}|t|^{\mathrm{i}-1} \\
\leq & \frac{\mathrm{i}(\lambda)_{\mu}(\mu)_{\mathrm{i}-1}(\gamma)_{\mathrm{i}-1}[\mathrm{i}(\sigma \eta+1)+\eta(\tau-\epsilon)-1]}{\eta(\tau-\epsilon+\sigma)(\lambda+\gamma)_{\mu}(\mu+\lambda+\gamma)_{\mathrm{i}-1}(\mathrm{i}-1) !},
\end{aligned}
$$

or equivalently

$$
|t| \leq\left\{\frac{\mathrm{i}(1-m)[\mathrm{i}(\sigma \eta+1)+\eta(\tau-\epsilon)-1]}{\eta(\mathrm{i}-m)(\tau-\epsilon+\sigma)}\right\}^{\frac{1}{\mathrm{j}-1}},
$$

this given the desired result.

Theorem 2.6. If $\mathfrak{P} \wp_{\lambda, \gamma}^{\mu} \in \mathcal{F}(\sigma, \tau, \epsilon, \eta, \mu, \lambda, \gamma)$, then $\mathfrak{P} \wp_{\lambda, \gamma}^{\mu}$ will be convex of order $m(0 \leq m<1)$ in the disk $|t|<r_{2}$, where

$$
r_{2}=\inf _{\dot{i}}\left\{\frac{(1-m)[\mathrm{i}(\sigma \eta+1)+\eta(\tau-\epsilon)-1]}{\eta(\mathrm{i}-m)(\tau-\epsilon+\sigma)}\right\}^{\frac{1}{\mathrm{i}-1}}, \quad(\mathrm{i} \geq 2) .
$$

Proof. It is enough to show that 


$$
\left|\frac{t\left(\mathfrak{P} \wp_{\lambda, \gamma}^{\mu}(t)\right)^{\prime \prime}}{\left(\mathfrak{P} \wp_{\lambda, \gamma}^{\mu}(t)\right)^{\prime}}\right| \leq 1-m \quad \text { for }|t|<r_{2}
$$

The result follows by application of arguments similar to the proof of Theorem 2.5.

Now, we consider the functions $\mathfrak{U}_{n}(n=1,2)$ which defined by

$$
\mathfrak{U}_{n}(t)=t-\sum_{\mathrm{i}=2}^{\infty} a_{\mathrm{i}, n} t^{\mathrm{i}}, \quad\left(a_{\mathrm{i}, n} \geq 0, n=1,2\right) .
$$

Then, by making use of (1.5), we have

$$
\mathfrak{P} \wp_{\lambda, \gamma_{n}}^{\mu}(t)=t-\sum_{\mathrm{i}=2}^{\infty} \frac{(\lambda)_{\mu}(\mu)_{\mathrm{i}-1}(\gamma)_{\mathrm{i}-1}}{(\lambda+\gamma)_{\mu}(\mu+\lambda+\gamma)_{\mathrm{i}-1}(\mathrm{i}-1) !} a_{\mathrm{i}, n} t^{\mathrm{i}}, \quad\left(a_{\mathrm{i}, n} \geq 0, n=1,2\right) .
$$

Theorem 2.7. Assume that the functions $\mathfrak{P} \wp_{\lambda, \gamma_{n}}^{\mu}(n=1,2)$ defined by (2.11) be in the family $\mathcal{F}(\sigma, \tau, \epsilon, \eta, \mu, \lambda, \gamma)$. Then $\mathfrak{P} \wp_{\lambda, \gamma_{1}}^{\mu} * \mathfrak{P} \wp_{\lambda, \gamma_{2}}^{\mu} \in \mathcal{F}(\sigma, \tau, \xi, \eta, \mu, \lambda, \gamma)$, where

$$
\begin{aligned}
& \xi \leq \frac{\mathrm{i}(\tau+\sigma)(\lambda)_{\mu}(\mu)_{\mathrm{i}-1}(\gamma)_{\mathrm{i}-1}[\mathrm{i}(\sigma \eta+1)+\eta(\tau-\epsilon)-1]^{2}}{\mathrm{i}(\lambda)_{\mu}(\mu)_{\mathrm{i}-1}(\gamma)_{\mathrm{i}-1}[\mathrm{i}(\sigma \eta+1)+\eta(\tau-\epsilon)-1]^{2}-\eta^{2}(\tau-\epsilon+\sigma)^{2}(\lambda+\gamma)_{\mu}(\mu+\lambda+\gamma)_{\mathrm{i}-1}(\mathrm{i}-1) !} \\
&-\frac{\eta(\tau-\epsilon+\sigma)^{2}(\lambda+\gamma)_{\mu}(\mu+\lambda+\gamma)_{\mathrm{i}-1}(\mathrm{i}-1) ![\mathrm{i}(\sigma \eta+1)+\eta \tau-1]}{\mathrm{i}(\lambda)_{\mu}(\mu)_{\mathrm{i}-1}(\gamma)_{\mathrm{i}-1}[\mathrm{i}(\sigma \eta+1)+\eta(\tau-\epsilon)-1]^{2}-\eta^{2}(\tau-\epsilon+\sigma)^{2}(\lambda+\gamma)_{\mu}(\mu+\lambda+\gamma)_{\mathrm{i}-1}(\mathrm{i}-1) !}
\end{aligned}
$$

Proof. We want find the largest $\xi$ such that

$$
\sum_{\mathrm{i}=2}^{\infty} \frac{\mathrm{i}(\lambda)_{\mu}(\mu)_{\mathrm{i}-1}(\gamma)_{\mathrm{i}-1}[\mathrm{i}(\sigma \eta+1)+\eta(\tau-\xi)-1]}{(\lambda+\gamma)_{\mu}(\mu+\lambda+\gamma)_{\mathrm{i}-1}(\mathrm{j}-1) ! \eta(\tau-\xi+\sigma)} a_{\mathrm{i}, 1} a_{\mathrm{i}, 2} \leq 1 .
$$

Since $\mathfrak{P} \rho_{\lambda, \gamma_{n}}^{\mu} \in \mathcal{F}(\sigma, \tau, \epsilon, \eta, \mu, \lambda, \gamma)(n=1,2)$, we get

$$
\sum_{\mathrm{i}=2}^{\infty} \frac{\mathrm{i}(\lambda)_{\mu}(\mu)_{\mathrm{i}-1}(\gamma)_{\mathrm{i}-1}[\mathrm{i}(\sigma \eta+1)+\eta(\tau-\epsilon)-1]}{(\lambda+\gamma)_{\mu}(\mu+\lambda+\gamma)_{\mathrm{i}-1}(\mathrm{i}-1) ! \eta(\tau-\epsilon+\sigma)} a_{\mathrm{i}, n} \leq 1, \quad(n=1,2) .
$$

By applying "Cauchy-Schwarz inequality", we conclude that

$$
\sum_{\mathrm{i}=2}^{\infty} \frac{\mathrm{i}(\lambda)_{\mu}(\mu)_{\mathrm{i}-1}(\gamma)_{\mathrm{i}-1}[\mathrm{i}(\sigma \eta+1)+\eta(\tau-\epsilon)-1]}{(\lambda+\gamma)_{\mu}(\mu+\lambda+\gamma)_{\mathrm{i}-1}(\mathrm{i}-1) ! \eta(\tau-\epsilon+\sigma)} \sqrt{a_{\mathrm{i}, 1} a_{\mathrm{i}, 2}} \leq 1 .
$$


We want only to show that

$$
\begin{aligned}
& \frac{\mathrm{i}(\lambda)_{\mu}(\mu)_{\mathrm{i}-1}(\gamma)_{\mathrm{i}-1}[\mathrm{i}(\sigma \eta+1)+\eta(\tau-\xi)-1]}{(\lambda+\gamma)_{\mu}(\mu+\lambda+\gamma)_{\mathrm{i}-1}(\mathrm{i}-1) ! \eta(\tau-\xi+\sigma)} a_{\mathrm{i}, 1} a_{\mathrm{i}, 2} \\
\leq & \frac{\mathrm{i}(\lambda)_{\mu}(\mu)_{\mathrm{i}-1}(\gamma)_{\mathrm{i}-1}[\mathrm{i}(\sigma \eta+1)+\eta(\tau-\epsilon)-1]}{(\lambda+\gamma)_{\mu}(\mu+\lambda+\gamma)_{\mathrm{i}-1}(\mathrm{j}-1) ! \eta(\tau-\epsilon+\sigma)} \sqrt{a_{\mathrm{i}, 1} a_{\mathrm{i}, 2}} .
\end{aligned}
$$

This equivalently to

$$
\sqrt{a_{\mathrm{i}, 1} a_{\mathrm{i}, 2}} \leq \frac{(\tau-\xi+\sigma)[\mathrm{i}(\sigma \eta+1)+\eta(\tau-\epsilon)-1]}{(\tau-\epsilon+\sigma)[\mathrm{i}(\sigma \eta+1)+\eta(\tau-\xi)-1]} .
$$

From (2.12), we have

$$
\sqrt{a_{\mathrm{j}, 1} a_{\mathrm{i}, 2}} \leq \frac{(\lambda+\gamma)_{\mu}(\mu+\lambda+\gamma)_{\mathrm{i}-1}(\mathrm{i}-1) ! \eta(\tau-\epsilon+\sigma)}{\mathrm{i}(\lambda)_{\mu}(\mu)_{\mathrm{i}-1}(\gamma)_{\mathrm{i}-1}[\mathrm{i}(\sigma \eta+1)+\eta(\tau-\epsilon)-1]} .
$$

Thus, it is sufficient to show that

$$
\frac{(\lambda+\gamma)_{\mu}(\mu+\lambda+\gamma)_{\mathrm{i}-1}(\mathrm{i}-1) ! \eta(\tau-\epsilon+\sigma)}{\mathrm{i}(\lambda)_{\mu}(\mu)_{\mathrm{i}-1}(\gamma)_{\mathrm{i}-1}[\mathrm{i}(\sigma \eta+1)+\eta(\tau-\epsilon)-1]} \leq \frac{(\tau-\xi+\sigma)[\mathrm{i}(\sigma \eta+1)+\eta(\tau-\epsilon)-1]}{(\tau-\epsilon+\sigma)[\mathrm{i}(\sigma \eta+1)+\eta(\tau-\xi)-1]}
$$

which implies to

$$
\begin{gathered}
\xi \leq \frac{\mathrm{i}(\tau+\sigma)(\lambda)_{\mu}(\mu)_{\mathrm{i}-1}(\gamma)_{\mathrm{i}-1}[\mathrm{i}(\sigma \eta+1)+\eta(\tau-\epsilon)-1]^{2}}{\mathrm{i}(\lambda)_{\mu}(\mu)_{\mathrm{i}-1}(\gamma)_{\mathrm{i}-1}[\mathrm{i}(\sigma \eta+1)+\eta(\tau-\epsilon)-1]^{2}-\eta^{2}(\tau-\epsilon+\sigma)^{2}(\lambda+\gamma)_{\mu}(\mu+\lambda+\gamma)_{\mathrm{i}-1}(\mathrm{j}-1) !} \\
-\frac{\eta(\tau-\epsilon+\sigma)^{2}(\lambda+\gamma)_{\mu}(\mu+\lambda+\gamma)_{\mathrm{i}-1}(\mathrm{i}-1) ![\mathrm{i}(\sigma \eta+1)+\eta \tau-1]}{\mathrm{i}(\lambda)_{\mu}(\mu)_{\mathrm{i}-1}(\gamma)_{\mathrm{i}-1}[\mathrm{i}(\sigma \eta+1)+\eta(\tau-\epsilon)-1]^{2}-\eta^{2}(\tau-\epsilon+\sigma)^{2}(\lambda+\gamma)_{\mu}(\mu+\lambda+\gamma)_{\mathrm{i}-1}(\mathrm{j}-1) !} .
\end{gathered}
$$

Theorem 2.8. Assume that the functions $\mathfrak{P} \rho_{\lambda, \gamma_{n}}^{\mu}(n=1,2)$ defined by (2.11) be in the family $\mathcal{F}(\sigma, \tau, \epsilon, \eta, \mu, \lambda, \gamma)$. Then the function $\mathcal{K}$ defined by

$$
\mathcal{K}(t)=t-\sum_{\mathrm{i}=2}^{\infty} \frac{(\lambda)_{\mu}(\mu)_{\mathrm{i}-1}(\gamma)_{\mathrm{i}-1}}{(\lambda+\gamma)_{\mu}(\mu+\lambda+\gamma)_{\mathrm{i}-1}(\mathrm{i}-1) !}\left(a_{\mathrm{i}, 1}^{2}+a_{\mathrm{i}, 2}^{2}\right) t^{\mathrm{i}}
$$

belong to the family $\mathcal{F}(\sigma, \tau, \zeta, \eta, \mu, \lambda, \gamma)$, where

$$
\zeta \leq \frac{\mathrm{i}(\tau+\sigma)[\mathrm{i}(\sigma \eta+1)+\eta(\tau-\epsilon)-1]^{2}-2 \eta(\tau-\epsilon+\sigma)^{2}[\mathrm{i}(\sigma \eta+1)+\eta \tau-1]}{\mathrm{i}[\mathrm{i}(\sigma \eta+1)+\eta(\tau-\epsilon)-1]^{2}-2 \eta^{2}(\tau-\epsilon+\sigma)^{2}} .
$$

Proof. We need find the largest $\zeta$ such that 


$$
\sum_{\mathrm{i}=2}^{\infty} \frac{\mathrm{i}\left((\lambda)_{\mu}\right)^{2}\left((\mu)_{\mathrm{i}-1}\right)^{2}\left((\gamma)_{\mathrm{i}-1}\right)^{2}[\mathrm{i}(\sigma \eta+1)+\eta(\tau-\zeta)-1]}{\left((\lambda+\gamma)_{\mu}\right)^{2}\left((\mu+\lambda+\gamma)_{\mathrm{i}-1}\right)^{2}((\mathrm{i}-1) !)^{2} \eta(\tau-\zeta+\sigma)}\left(a_{\mathrm{j}, 1}^{2}+a_{\mathrm{j}, 2}^{2}\right) \leq 1 .
$$

Since $\mathfrak{P} \wp_{\lambda, \gamma_{n}}^{\mu} \in \mathcal{F}(\sigma, \tau, \epsilon, \eta, \mu, \lambda, \gamma)(n=1,2)$, we get

$$
\begin{aligned}
& \sum_{\mathrm{i}=2}^{\infty}\left(\frac{\mathrm{i}(\lambda)_{\mu}(\mu)_{\mathrm{i}-1}(\gamma)_{\mathrm{i}-1}[\mathrm{i}(\sigma \eta+1)+\eta(\tau-\epsilon)-1]}{(\lambda+\gamma)_{\mu}(\mu+\lambda+\gamma)_{\mathrm{i}-1}(\mathrm{i}-1) ! \eta(\tau-\epsilon+\sigma)}\right)^{2} a_{\mathrm{i}, 1}^{2} \\
\leq & \left(\sum_{\mathrm{i}=2}^{\infty} \frac{\mathrm{i}(\lambda)_{\mu}(\mu)_{\mathrm{i}-1}(\gamma)_{\mathrm{i}-1}[\mathrm{i}(\sigma \eta+1)+\eta(\tau-\epsilon)-1]}{(\lambda+\gamma)_{\mu}(\mu+\lambda+\gamma)_{\mathrm{i}-1}(\mathrm{i}-1) ! \eta(\tau-\epsilon+\sigma)} a_{\mathrm{i}, 1}\right)^{2} \leq 1
\end{aligned}
$$

and

$$
\begin{aligned}
& \sum_{\mathrm{i}=2}^{\infty}\left(\frac{\mathrm{i}(\lambda)_{\mu}(\mu)_{\mathrm{i}-1}(\gamma)_{\mathrm{i}-1}[\mathrm{i}(\sigma \eta+1)+\eta(\tau-\epsilon)-1]}{(\lambda+\gamma)_{\mu}(\mu+\lambda+\gamma)_{\mathrm{i}-1}(\mathrm{i}-1) ! \eta(\tau-\epsilon+\sigma)}\right)^{2} a_{\mathrm{i}, 2}^{2} \\
\leq & \left(\sum_{\mathrm{i}=2}^{\infty} \frac{\mathrm{i}(\lambda)_{\mu}(\mu)_{\mathrm{i}-1}(\gamma)_{\mathrm{i}-1}[\mathrm{i}(\sigma \eta+1)+\eta(\tau-\epsilon)-1]}{(\lambda+\gamma)_{\mu}(\mu+\lambda+\gamma)_{\mathrm{i}-1}(\mathrm{i}-1) ! \eta(\tau-\epsilon+\sigma)} a_{\mathrm{i}, 2}\right)^{2} \leq 1 .
\end{aligned}
$$

Combining the inequalities (2.14) and (2.15), gives

$$
\sum_{\mathrm{i}=2}^{\infty} \frac{1}{2}\left(\frac{\mathrm{i}(\lambda)_{\mu}(\mu)_{\mathrm{i}-1}(\gamma)_{\mathrm{i}-1}[\mathrm{i}(\sigma \eta+1)+\eta(\tau-\epsilon)-1]}{(\lambda+\gamma)_{\mu}(\mu+\lambda+\gamma)_{\mathrm{i}-1}(\mathrm{i}-1) ! \eta(\tau-\epsilon+\sigma)}\right)^{2}\left(a_{\mathrm{i}, 1}^{2}+a_{\mathrm{i}, 2}^{2}\right) \leq 1 .
$$

But, $\mathcal{K} \in \mathcal{F}(\sigma, \tau, \zeta, \eta, \mu, \lambda, \gamma)$ if and only if

$$
\sum_{\mathrm{i}=2}^{\infty} \frac{\mathrm{i}\left((\lambda)_{\mu}\right)^{2}\left((\mu)_{\mathrm{i}-1}\right)^{2}\left((\gamma)_{\mathrm{i}-1}\right)^{2}[\mathrm{i}(\sigma \eta+1)+\eta(\tau-\zeta)-1]}{\left((\lambda+\gamma)_{\mu}\right)^{2}\left((\mu+\lambda+\gamma)_{\mathrm{i}-1}\right)^{2}((\mathrm{i}-1) !)^{2} \eta(\tau-\zeta+\sigma)}\left(a_{\mathrm{i}, 1}^{2}+a_{\mathrm{j}, 2}^{2}\right) \leq 1 .
$$

The inequality (2.16) will be satisfied if

$$
\begin{aligned}
& \frac{\mathrm{i}\left((\lambda)_{\mu}\right)^{2}\left((\mu)_{\mathrm{i}-1}\right)^{2}\left((\gamma)_{\mathrm{i}-1}\right)^{2}[\mathrm{i}(\sigma \eta+1)+\eta(\tau-\zeta)-1]}{\left((\lambda+\gamma)_{\mu}\right)^{2}\left((\mu+\lambda+\gamma)_{\mathrm{i}-1}\right)^{2}((\mathrm{i}-1) !)^{2} \eta(\tau-\zeta+\sigma)} \\
\leq & \frac{\mathrm{i}^{2}\left((\lambda)_{\mu}\right)^{2}\left((\mu)_{\mathrm{i}-1}\right)^{2}\left((\gamma)_{\mathrm{i}-1}\right)^{2}[\mathrm{i}(\sigma \eta+1)+\eta(\tau-\epsilon)-1]^{2}}{2\left((\lambda+\gamma)_{\mu}\right)^{2}\left((\mu+\lambda+\gamma)_{\mathrm{i}-1}\right)^{2}((\mathrm{i}-1) !)^{2} \eta^{2}(\tau-\epsilon+\sigma)^{2}}, \quad(\mathrm{i}=2,3, \ldots)
\end{aligned}
$$


so that

$$
\zeta \leq \frac{\mathrm{i}(\tau+\sigma)[\mathrm{i}(\sigma \eta+1)+\eta(\tau-\epsilon)-1]^{2}-2 \eta(\tau-\epsilon+\sigma)^{2}[\mathrm{i}(\sigma \eta+1)+\eta \tau-1]}{\mathrm{i}[\mathrm{i}(\sigma \eta+1)+\eta(\tau-\epsilon)-1]^{2}-2 \eta^{2}(\tau-\epsilon+\sigma)^{2}} .
$$

Theorem 2.9. Assume that the functions $\mathfrak{P} \rho_{\lambda, \gamma_{n}}^{\mu}(n=1,2)$ defined by (2.11) be in the family $\mathcal{F}(\sigma, \tau, \epsilon, \eta, \mu, \lambda, \gamma)$ and $\eta \sigma \geq-1$. Then the function $\mathcal{K}$ defined by (2.13) belongs to the family $\mathcal{F}(\sigma, \tau, \epsilon, \eta, \mu, \lambda, \gamma)$.

Proof. Since $\mathfrak{P} \wp_{\lambda, \gamma_{1}}^{\mu} \in \mathcal{F}(\sigma, \tau, \epsilon, \eta, \mu, \lambda, \gamma)$, we have

$$
\sum_{\mathrm{i}=2}^{\infty} \frac{\mathrm{i}(\lambda)_{\mu}(\mu)_{\mathrm{i}-1}(\gamma)_{\mathrm{i}-1}[\mathrm{i}(\sigma \eta+1)+\eta(\tau-\epsilon)-1]}{(\lambda+\gamma)_{\mu}(\mu+\lambda+\gamma)_{\mathrm{i}-1}(\mathrm{i}-1) ! \eta(\tau-\epsilon+\sigma)} a_{\mathrm{j}, 1} \leq 1
$$

and so

$$
\begin{aligned}
& \sum_{\mathrm{i}=2}^{\infty}\left(\frac{\mathrm{i}(\lambda)_{\mu}(\mu)_{\mathrm{i}-1}(\gamma)_{\mathrm{i}-1}[\mathrm{i}(\sigma \eta+1)+\eta(\tau-\epsilon)-1]}{(\lambda+\gamma)_{\mu}(\mu+\lambda+\gamma)_{\mathrm{i}-1}(\mathrm{i}-1) ! \eta(\tau-\epsilon+\sigma)}\right)^{2} a_{\mathrm{i}, 1}^{2} \\
\leq & \left(\sum_{\mathrm{i}=2}^{\infty} \frac{\mathrm{i}(\lambda)_{\mu}(\mu)_{\mathrm{i}-1}(\gamma)_{\mathrm{i}-1}[\mathrm{i}(\sigma \eta+1)+\eta(\tau-\epsilon)-1]}{(\lambda+\gamma)_{\mu}(\mu+\lambda+\gamma)_{\mathrm{i}-1}(\mathrm{i}-1) ! \eta(\tau-\epsilon+\sigma)} a_{\mathrm{i}, 1}\right)^{2} \leq 1 .
\end{aligned}
$$

Similarly, since $\mathfrak{P} \wp_{\lambda, \gamma_{2}}^{\mu} \in \mathcal{F}(\sigma, \tau, \epsilon, \eta, \mu, \lambda, \gamma)$, we have

$$
\sum_{\mathrm{i}=2}^{\infty}\left(\frac{\mathrm{i}(\lambda)_{\mu}(\mu)_{\mathrm{i}-1}(\gamma)_{\mathrm{i}-1}[\mathrm{i}(\sigma \eta+1)+\eta(\tau-\epsilon)-1]}{(\lambda+\gamma)_{\mu}(\mu+\lambda+\gamma)_{\mathrm{i}-1}(\mathrm{i}-1) ! \eta(\tau-\epsilon+\sigma)}\right)^{2} a_{\mathrm{i}, 2}^{2} \leq 1 .
$$

Hence

$$
\sum_{\mathrm{i}=2}^{\infty} \frac{1}{2}\left(\frac{\mathrm{i}(\lambda)_{\mu}(\mu)_{\mathrm{i}-1}(\gamma)_{\mathrm{i}-1}[\mathrm{i}(\sigma \eta+1)+\eta(\tau-\epsilon)-1]}{(\lambda+\gamma)_{\mu}(\mu+\lambda+\gamma)_{\mathrm{i}-1}(\mathrm{j}-1) ! \eta(\tau-\epsilon+\sigma)}\right)^{2}\left(a_{\mathrm{i}, 1}^{2}+a_{\mathrm{i}, 2}^{2}\right) \leq 1 .
$$

In light of Theorem 2.1, it is enough to show that

$$
\sum_{\mathrm{i}=p+1}^{\infty} \frac{\mathrm{i}\left((\lambda)_{\mu}\right)^{2}\left((\mu)_{\mathrm{i}-1}\right)^{2}\left((\gamma)_{\mathrm{i}-1}\right)^{2}[\mathrm{i}(\sigma \eta+1)+\eta(\tau-\zeta)-1]}{\left((\lambda+\gamma)_{\mu}\right)^{2}\left((\mu+\lambda+\gamma)_{\mathrm{i}-1}\right)^{2}((\mathrm{i}-1) !)^{2} \eta(\tau-\zeta+\sigma)}\left(a_{\mathrm{i}, 1}^{2}+a_{\mathrm{i}, 2}^{2}\right) \leq 1 .
$$

Thus the inequality (2.17) will be satisfied if 


$$
\begin{aligned}
& \frac{\mathrm{i}\left((\lambda)_{\mu}\right)^{2}\left((\mu)_{\mathrm{i}-1}\right)^{2}\left((\gamma)_{\mathrm{i}-1}\right)^{2}[\mathrm{i}(\sigma \eta+1)+\eta(\tau-\epsilon)-1]}{\left((\lambda+\gamma)_{\mu}\right)^{2}\left((\mu+\lambda+\gamma)_{\mathrm{i}-1}\right)^{2}((\mathrm{i}-1) !)^{2} \eta(\tau-\epsilon+\sigma)} \\
\leq & \frac{\mathfrak{i}^{2}\left((\lambda)_{\mu}\right)^{2}\left((\mu)_{\mathrm{i}-1}\right)^{2}\left((\gamma)_{\mathrm{i}-1}\right)^{2}[\mathrm{i}(\sigma \eta+1)+\eta(\tau-\epsilon)-1]^{2}}{2\left((\lambda+\gamma)_{\mu}\right)^{2}\left((\mu+\lambda+\gamma)_{\mathrm{i}-1}\right)^{2}((\mathrm{i}-1) !)^{2} \eta^{2}(\tau-\epsilon+\sigma)^{2}}, \quad(\mathrm{i}=2,3, \ldots)
\end{aligned}
$$

or if

$$
\mathrm{i}[\mathrm{i}(\sigma \eta+1)+\eta(\tau-\epsilon)-1]-2 \eta(\tau-\epsilon+\sigma) \geq 0, \quad(\mathrm{j}=2,3, \ldots) .
$$

The left hand side of (2.18) is increasing function of $\mathfrak{j}$, hence (2.18) is satisfied for all $\mathfrak{j}$ if $\eta \sigma \geq-1$. This completes the proof.

In the next theorems, we discuss some subordination results for the function $\mathfrak{P} \rho_{\lambda, \gamma}^{\mu}$.

Theorem 2.10. Let $\mathfrak{Q}$ be univalent in $\mathfrak{D}$ with $\mathfrak{L}(t) \neq 0(t \in \mathfrak{D})$ and assume that $\mathfrak{L}$ fulfills

$$
\mathfrak{R}\left\{1+\frac{\mathfrak{E M}}{\varrho}+\frac{\mathfrak{N}(\mathfrak{M}+1)}{\varrho} \mathfrak{L}(t)+(\mathfrak{M}-1) \frac{t \mathfrak{Q}^{\prime}(t)}{\mathfrak{L}(t)}+\frac{t \mathfrak{Q}^{\prime \prime}(t)}{\mathfrak{L}^{\prime}(t)}\right\}>0,
$$

where $\mathfrak{E}, \aleph, \mathfrak{M} \in \mathbb{C}, \varrho \in \mathbb{C} \backslash\{0\}$ and $t \in \mathfrak{D}$.

Suppose that $t(\mathfrak{L}(t))^{\mathfrak{M}-1} \mathfrak{Q}^{\prime}(t)$ is starlike univalent in $\mathfrak{D}$. If a function $\mathfrak{P} \wp_{\lambda, \gamma}^{\mu}$ fulfills the subordination

$$
\begin{gathered}
\mathfrak{E}\left(\frac{t\left(\mathfrak{P} \wp_{\lambda, \gamma}^{\mu}(t)\right)^{\prime}}{\mathfrak{P} \wp_{\lambda, \gamma}^{\mu}(t)}\right)^{\beta \mathfrak{M}}+\aleph\left(\frac{t\left(\mathfrak{P} \wp_{\lambda, \gamma}^{\mu}(t)\right)^{\prime}}{\mathfrak{P} \wp_{\lambda, \gamma}^{\mu}(t)}\right)^{\beta(\mathfrak{M}+1)}+\varrho \beta\left(\frac{t\left(\mathfrak{P} \wp_{\lambda, \gamma}^{\mu}(t)\right)^{\prime}}{\mathfrak{P} \wp_{\lambda, \gamma}^{\mu}(t)}\right)^{\beta \mathfrak{M}} \\
\times\left(1+\frac{t\left(\mathfrak{P} \wp_{\lambda, \gamma}^{\mu}(t)\right)^{\prime \prime}}{\left(\mathfrak{P} \wp_{\lambda, \gamma}^{\mu}(t)\right)^{\prime}}-\frac{t\left(\mathfrak{P} \wp_{\lambda, \gamma}^{\mu}(t)\right)^{\prime}}{\mathfrak{P} \wp_{\lambda, \gamma}^{\mu}(t)}\right) \\
<(\mathfrak{E}+א \mathfrak{L}(t))(\mathfrak{L}(t))^{\mathfrak{M}}+\varrho t(\mathfrak{L}(t))^{\mathfrak{M}-1} \mathfrak{L}^{\prime}(t)
\end{gathered}
$$

then$$
\left(\frac{t\left(\mathfrak{P} \wp_{\lambda, \gamma}^{\mu}(t)\right)^{\prime}}{\mathfrak{P} \wp_{\lambda, \gamma}^{\mu}(t)}\right)^{\ell} \prec \mathfrak{L}(t) \quad(\ell>0, t \in \mathfrak{D})
$$

and $\mathfrak{L}$ is the best dominant. 
Proof. Assume that $\mathcal{T}$ be defined by

$$
\mathcal{T}(t)=\left(\frac{t\left(\mathfrak{P} \wp_{\lambda, \gamma}^{\mu}(t)\right)^{\prime}}{\mathfrak{P} \wp_{\lambda, \gamma}^{\mu}(t)}\right)^{\ell}=\left(\frac{1-\sum_{\mathrm{j}=2}^{\infty} \mathrm{i} \frac{(\lambda)_{\mu}(\mu)_{\mathrm{i}-1}(\gamma)_{\mathrm{i}-1}}{(\lambda+\gamma)_{\mu}(\mu+\lambda+\gamma)_{\mathrm{i}-1}(\mathrm{i}-1) !} a_{\mathrm{i}} t^{\mathrm{i}-1}}{1-\sum_{\mathrm{j} n=2}^{\infty} \frac{(\lambda)_{\mu}(\mu)_{\mathrm{i}-1}(\gamma)_{\mathrm{i}-1}}{(\lambda+\gamma)_{\mu}(\mu+\lambda+\gamma)_{\mathrm{i}-1}(\mathrm{i}-1) !} a_{\mathrm{i}} t^{\mathrm{i}-1}}\right)^{\ell} .
$$

It can be easily observed that $\mathcal{T}$ is holomorphic in $\mathfrak{D}$.

By setting

$$
\vartheta(w)=(\mathfrak{E}+\aleph w) w^{\mathfrak{M}} \text { and } \varphi(w)=\varrho w^{\mathfrak{M}-1}, w \neq 0,
$$

we find that $\vartheta(w)$ is holomorphic in $\mathbb{C}, \varphi(w)$ is holomorphic in $\mathbb{C} \backslash\{0\}$ and that $\varphi(w) \neq$ $0, w \in \mathbb{C} \backslash\{0\}$. Also, we obtain

$$
\mathcal{Q}(t)=t \mathfrak{L}^{\prime}(t) \varphi(\mathfrak{L}(t))=\varrho t(\mathfrak{L}(t))^{\mathfrak{M}-1} \mathfrak{L}^{\prime}(t)
$$

and

$$
\mathfrak{H}(t)=\vartheta(\mathfrak{L}(t))+\mathcal{Q}(t)=(\epsilon+\mathcal{N} \mathbb{L}(t))(\mathfrak{L}(t))^{\mathfrak{M}}+\varrho t(\mathfrak{L}(t))^{\mathfrak{M}-1} \mathfrak{L}^{\prime}(t) .
$$

It is obvious that $Q(t)$ is starlike univalent in $\mathfrak{D}$ and from (2.19), we conclude that

$$
\mathfrak{R}\left\{\frac{t \mathfrak{S}^{\prime}(t)}{\mathcal{Q}(t)}\right\}=\mathfrak{R}\left\{1+\frac{\mathfrak{E M}}{\varrho}+\frac{\aleph(\mathfrak{M}+1)}{\varrho} \mathfrak{L}(t)+(\mathfrak{M}-1) \frac{t \mathfrak{L}^{\prime}(t)}{\mathfrak{L}(t)}+\frac{t \mathfrak{L}^{\prime \prime}(t)}{\mathfrak{L}^{\prime}(t)}\right\}>0 .
$$

By some calculation, we have

$$
\begin{aligned}
& (\mathfrak{E}+\aleph \mathcal{T}(t))(\mathcal{T}(t))^{\mathfrak{M}}+\varrho t(\mathcal{T}(t))^{\mathfrak{M}-1} \mathcal{T}^{\prime}(t) \\
= & \mathfrak{E}\left(\frac{t\left(\mathfrak{P} \wp_{\lambda, \gamma}^{\mu}(t)\right)^{\prime}}{\mathfrak{P} \wp_{\lambda, \gamma}^{\mu}(t)}\right)^{\ell \mathfrak{M}}+\aleph\left(\frac{t\left(\mathfrak{P} \wp_{\lambda, \gamma}^{\mu}(t)\right)^{\prime}}{\mathfrak{P} \wp_{\lambda, \gamma}^{\mu}(t)}\right)^{\ell(\mathfrak{M}+1)} \\
& +\varrho \ell\left(\frac{t\left(\mathfrak{P} \wp_{\lambda, \gamma}^{\mu}(t)\right)^{\prime}}{\mathfrak{P} \wp_{\lambda, \gamma}^{\mu}(t)}\right)^{\ell \mathfrak{M}}\left(1+\frac{t\left(\mathfrak{P} \wp_{\lambda, \gamma}^{\mu}(t)\right)^{\prime \prime}}{\left(\mathfrak{P} \wp_{\lambda, \gamma}^{\mu}(t)\right)^{\prime}}-\frac{t\left(\mathfrak{P} \wp_{\lambda, \gamma}^{\mu}(t)\right)^{\prime}}{\mathfrak{P} \wp_{\lambda, \gamma}^{\mu}(t)}\right) .
\end{aligned}
$$

From (2.20) and (2.22), we find that

$$
\begin{aligned}
& (\mathfrak{E}+\kappa \mathcal{T}(t))(\mathcal{T}(t))^{\mathfrak{M}}+\varrho t(\mathcal{T}(t))^{\mathfrak{M}-1} \mathcal{T}^{\prime}(t) \\
\prec & (\mathfrak{E}+\kappa \mathfrak{L}(t))(\mathfrak{L}(t))^{\mathfrak{M}}+\varrho t(\mathfrak{L}(t))^{\mathfrak{M}-1} \mathfrak{L}^{\prime}(t) .
\end{aligned}
$$


Hence, by Lemma 1.1, we conclude that $\mathcal{T}(t) \prec \mathfrak{L}(t)$. From (2.21), we have the result.

Theorem 2.11. Let $u, v \in \mathbb{C}$ and $\mathfrak{I}$ be convex and univalent in $\mathfrak{D}$ with $\mathfrak{I}(0)=1$ and $\mathfrak{R}\{u \mathfrak{S}(t)+v\}>0,(t \in \mathfrak{D})$. If a function $\mathfrak{B} \wp_{\lambda, \gamma}^{\mu}$ fulfills the subordination

$$
\frac{1+v+\frac{t\left(\mathfrak{P}_{\lambda, \gamma}^{\mu}(t)\right)^{\prime \prime}}{\left(\mathfrak{P} \delta_{\lambda, \gamma}^{\mu}(t)\right)^{\prime}}+(u-1) \frac{t\left(\mathfrak{P}_{\lambda, \gamma}^{\mu}(t)\right)^{\prime}}{\mathfrak{P} \delta_{\lambda, \gamma}^{\mu}(t)}}{u+v \frac{\mathfrak{P} \delta_{\lambda, \gamma}^{\mu}(t)}{t\left(\mathfrak{P}_{\lambda, \gamma}^{\mu}(t)\right)^{\prime}}}<\mathfrak{J}(t),
$$

then

$$
\frac{t\left(\mathfrak{P} \wp_{\lambda, \gamma}^{\mu}(t)\right)^{\prime}}{\mathfrak{P} \rho_{\lambda, \gamma}^{\mu}(t)} \prec \mathfrak{S}(t)
$$

and $\mathfrak{I}$ is the best dominant.

Proof. Assume that $\mathfrak{Q}$ be defined by

$$
\mathfrak{L}(t)=\frac{t\left(\mathfrak{P} \wp_{\lambda, \gamma}^{\mu}(t)\right)^{\prime}}{\mathfrak{P} \wp_{\lambda, \gamma}^{\mu}(t)}=\frac{1-\sum_{\mathrm{j}=2}^{\infty} \mathrm{i} \frac{(\lambda)_{\mu}(\mu)_{\mathrm{i}-1}(\gamma)_{\mathrm{i}-1}}{(\lambda+\gamma)_{\mu}(\mu+\lambda+\gamma)_{\mathrm{i}-1}(\mathrm{i}-1) !} a_{\mathrm{i}} t^{\mathrm{i}-1}}{1-\sum_{\mathrm{j}=2}^{\infty} \frac{(\lambda)_{\mu}(\mu)_{\mathrm{i}-1}(\gamma)_{\mathrm{i}-1}}{(\lambda+\gamma)_{\mu}(\mu+\lambda+\gamma)_{\mathrm{i}-1}(\mathrm{i}-1) !} a_{\mathrm{i}} t^{\mathrm{i}-1}}
$$

It can be easily observed that $\mathfrak{L}$ is holomorphic in $\mathfrak{D}$ and $\mathfrak{L}(0)=1$.

After some computation, we obtain

$$
\mathfrak{L}(t)+\frac{t \mathfrak{L}^{\prime}(t)}{u \mathfrak{L}(t)+v}=\frac{1+v+\frac{t\left(\mathfrak{P} \wp_{\lambda, \gamma}^{\mu}(t)\right)^{\prime \prime}}{\left(\mathfrak{P} \wp_{\lambda, \gamma}^{\mu}(t)\right)^{\prime}}+(u-1) \frac{t\left(\mathfrak{P} \wp_{\lambda, \gamma}^{\mu}(t)\right)^{\prime}}{\mathfrak{P} \wp_{\lambda, \gamma}^{\mu}(t)}}{u+v \frac{\mathfrak{P} \delta_{\lambda, \gamma}^{\mu}(t)}{t\left(\mathfrak{P} \delta_{\lambda, \gamma}^{\mu}(t)\right)^{\prime}}} .
$$

From (2.23) and (2.25), we have

$$
\mathfrak{L}(t)+\frac{t \mathfrak{L}^{\prime}(t)}{u \mathfrak{L}(t)+v} \prec \mathfrak{I}(t) .
$$

Hence, by Lemma 1.2, we conclude that $\mathfrak{L}(t) \prec \mathfrak{I}(t)$. From (2.24), we have the result. 


\section{References}

[1] R. M. Ali, V. Ravichandran and N. Seenivasagan, Differential subordination and superordination of analytic functions defined by the Dziok-Srivastava operator, $J$. Franklin Inst. 347 (2010), 1762-1781. https://doi.org/10.1016/j.jfranklin.2010.08.009

[2] S. Altinkaya and S. Yalçin, Poisson distribution series for certain subclasses of starlike functions with negative coefficients, An. Univ. Oradea Fasc. Mat. 24(2) (2017), 5-8.

[3] A. A. Amer and M. Darus, Some properties of the class of univalent functions with negative coefficients, Applied Mathematics 3 (2012), 1851-1856.

https://doi.org/10.4236/am.2012.312251

[4] A. Amourah and M. Darus, Some properties of a new class of univalent functions involving a new generalized differential operator with negative coefficients, Indian J. Sci. Tech. 9(36) (2016), 1-7. https://doi.org/10.17485/ijst/2016/v9i36/97738

[5] M. K. Aouf, A. O. Mostafa and O.M. Algubouri, Some families of uniformly starlike and convex functions with negative coefficients, Acta Univ. Apulensis 45 (2016), 125-147.

[6] W. G. Atshan, A. K. Wanas and G. Murugusundaramoorthy, Properties and characteristics of certain subclass of multivalent prestartlike functions with negative coefficients, An. Univ. Oradea Fasc. Mat. 26 (2019), 17-24.

[7] A. A. Attiya and M. F. Yassen, Some subordination and superordination results associated with generalized Srivastava-Attiya operator, Filomat 31(1) (2017), 53-60. https://doi.org/10.2298/FIL1701053A

[8] N. E. Cho and H. M. Srivastava, Argument estimates of certain analytic functions defined by a class of multiplier transformations, Math. Comput. Modelling 37(1-2) (2003), 39-49. https://doi.org/10.1016/S0895-7177(03)80004-3

[9] P. Eenigenburg, S. S. Miller, P. T. Mocanu and M. O. Reade, On a Briot-Bouquet differential subordination, Rev. Roumaine Math. Pures Appl. 29 (1984), 567-573.

[10] S. M. El-Deeb, T. Bulboaca and J. Dziok, Pascal distribution series connected with certain subclasses of univalent functions, Kyungpook Math. J. 59(2) (2019), 301-314.

[11] S. P. Goyal, P. Goswami and H. Silverman, Subordination and superordination results for a class of analytic multivalent functions, Int. J. Math. Math. Sci. 2008, Art. ID 561638, 12pp. https://doi.org/10.1155/2008/561638

[12] A. W. Goodman, Univalent functions and non-analytic curves, Proc. Amer. Math. Soc. 8 (1975), 598-601. https://doi.org/10.1090/S0002-9939-1957-0086879-9 
[13] R. W. Ibrahim and M. Darus, On a univalent class involving differential subordination with applications, J. Math. Statistics 7(2) (2011), 137-143. https://doi.org/10.3844/jmssp.2011.137.143

[14] J. L. Liu, Sufficient conditions for strongly starlike functions involving the generalized Srivastava-Attiya operator, Integral Transforms Spec. Funct. 22 (2011), 79-90. https://doi.org/10.1080/10652469.2010.498110

[15] N. Magesh, N. B. Gatti and S. Mayilvaganan, Differential sandwich theorems for certain subclasses of analytic functions associated with operators, Acta Univ. Apulensis 27 (2011), 187-202.

[16] N. Magesh, G. Murugusundaramoorthy, T. Rosy and K. Muthunagai, Subordination and superordination for analytic functions associated with convolution structure, Int. J. Open Problems Complex Analysis 2(2) (2010), 67-81.

[17] A. O. Mostafa and M. K. Aouf, Sandwich theorems for certain subclasses of analytic functions defined by family of linear operators, J. Appl. Anal. 15(2) (2009), 269-280. https://doi.org/10.1515/JAA.2009.269

[18] G. Murugusundaramoorthy, Subordination results for spiral-like functions associated with the Srivastava-Attiya operator, Integral Transforms Spec. Funct. 23 (2012), 97-103. https://doi.org/10.1080/10652469.2011.562501

[19] G. Murugusundaramoorthy, Certain subclasses of univalent functions associated with a unication of the Srivastava-Attiya and Cho-Saigo-Srivastava operators, Novi Sad J. Math. 45 (2015), 59-76. https://doi.org/10.30755/NSJOM.2014.022

[20] G. Murugusundaramoorthy and T. Janani, Inclusion results associated with certain subclass of analytic functions involving calculus operator, TWMS J. Pure Appl. Math. 7 (2016), 63-75.

[21] G. Mugusundaramoorthy and N. Magesh, Subordination results and integral means for certain subclasses of analytic functions, Int. J. Pure Appl. Math. 47(1) (2008), 65-78.

[22] S. S. Miller and P. T. Mocanu, Subordinants of differential superordinations, Complex Variables 48(10) (2003), 815-826. https://doi.org/10.1080/02781070310001599322

[23] W. Nazeer, Q. Mehmood, S. M. Kang and A. U. Haq, An application of Binomial distribution series on certain analytic functions, J. Comput. Anal. Appl. 26 (2019), 11-17.

[24] S. Porwal and M. Kumar, A unified study on starlike and convex functions associated with Poisson distribution series, Afr. Mat. 27 (2016), 10-21.

https://doi.org/10.1007/s13370-016-0398-z

[25] J. K. Prajapat and R. K. Raina, Some applications of differential subordination for a 
general class of multivalently analytic functions involving a convolution structure, Math. J. Okayama Univ. 52 (2010), 147-158.

[26] S. Rahrovi, Subordination and superordination properties for convolution operator, Int. J. Nonlinear Anal. Appl. 6(2) (2015), 137-147.

[27] S. Ruscheweyh, Neighborhoods of univalent functions, Proc. Amer. Math. Soc. 81 (1981), 521-527. https://doi.org/10.1090/S0002-9939-1981-0601721-6

[28] H. Silverman, Univalent functions with negative coefficients, Proc. Amer. Math. Soc. 51(1) (1975), 109-116. https://doi.org/10.1090/S0002-9939-1975-0369678-0

[29] H. M. Srivastava and S. Gaboury, A new class of analytic functions defined by means of a generalization of the Srivastava-Attiya operator, J. Ineq. Appl. 2015 (2015), Article ID 39, 15 pp. https://doi.org/10.1186/s13660-015-0573-z

[30] H. M. Srivastava, A. S. Juma and H. M. Zayed, Univalence conditions for an integral operator defined by a generalization of the Srivastava-Attiya operator, Filomat 32 (2018), 2101-2114. https://doi.org/10.2298/FIL1806101S

[31] H. M. Srivastava, A. Prajapati and P. Gochhayat, Third order differential subordination and differential superordination results for analytic functions involving the SrivastavaAttiya operator, Appl. Math. Inform. Sci. 12 (2018), 469-481.

https://doi.org/10.18576/amis/120301

[32] S. R. Swamy, Inclusion properties of certain subclasses of analytic functions, Int. Math. Forum 7(36) (2012), 1751-1760.

[33] A. K. Wanas and J. A. Khuttar, Applications of Borel distribution series on analytic functions, Earthline Journal of Mathematical Sciences 4(1) (2020), 71-82. https://doi.org/10.34198/ejms.4120.7182

[34] Q.-H. Xu, H.-G. Xiao and H. M. Srivastava, Some applications of differential subordination and the Dziok-Srivastava convolution operator, Appl. Math. Comput. 230 (2014), 496-508. https://doi.org/10.1016/j.amc.2013.12.065

[35] Y. Yunus, A. B. Akbarally and S. A. Halim, Properties of a certain subclass of starlike functions defined by a generalized operator, Int. J. Appl. Math. 31(4) (2018), 597-611. https://doi.org/10.12732/ijam.v31i4.6 